Gerhard Litscher und die Mitglieder des EPU-(Eurasia Pacific Uninet-)Projektteams

\title{
HIGH-TECH AKUPUNKTUR FÜR DIE PRÄVENTION VON LEBENSSTIL BEDINGTEN ERKRANKUNGEN - EINE „SINO-AUSTRIAN“ KOOPERATION - FORTSETZUNG
}

\author{
Ein Projektbericht (Studiendesign) zur Teleakupunktur mit Wuhan in COVID-19-Pandemie-Zeiten
}

High-Tech Acupuncture for Prevention of Lifestyle Diseases -
A Sino-Austrian Cooperation - Continuation

A Project Report (Study Design) on Tele-Acupuncture with Wuhan in COVID-19-Pandemic-Times

\section{Zusammenfassung}

Dieses Projekt im Rahmen einer erfolgreichen chinesisch-österreichischen Zusammenarbeit im Bereich High-Tech-Akupunktur zwischen der Hubei-Universität für Chinesische Medizin in Wuhan und der Medizinischen Universität Graz befasst sich mit klinischen transkontinentalen Tele-Akupunkturmessungen bei gesunden Freiwilligen und PatientInnen mit Müdigkeit und Stress. Herzraten- (HR) und Herzratenvariabilitätsmessungen (HRV) sollten unter standardisierten Bedingungen in Wuhan, China, durchgeführt werden, und die Datenanalysen werden in Graz, Österreich, bewerkstelligt. Ähnlich wie bei früheren Messungen sollen die Elektrokardiogramme während der Akupunktur und/oder Moxibustion von HRV-Systemen aufgezeichnet werden. Die Werte während und nach der Stimulation der GesamtHRV, der Niederfrequenz (LF) und der Hochfrequenz (HF) sowie die des LF/HF-Verhältnisses werden mit den Ausgangswerten und Werten gesunder Freiwilliger verglichen. Die Ergebnisse können als solide Grundlage für die Weiterentwicklung von Akupunkturstimulations- und Moxibustionsgeräten zur ergänzenden Anwendung bei der Behandlung von Müdigkeit und Stresssymptomen dienen.

\section{Schlüsselwörter}

Akupunktur, Lebensstil-Erkrankungen, High-TechAkupunktur, chinesisch-österreichische Zusammenarbeit, Herzratenvariabilität, evidenzbasierte Komplementärmedizin

\section{Summary}

This project within a successful Sino-Austrian cooperation on high-tech acupuncture between Hubei University of Chinese Medicine in Wuhan and Medical University of Graz deals with clinical transcontinental tele acupuncture measurements in healthy volunteers and patients with fatigue and stress.

Heart rate (HR) and heart rate variability (HRV) measurements should be performed under standardized conditions in Wuhan, China, and the data analyses should be performed in Graz,Austria. Similar to previous measurements, the electrocardiograms should be recorded during acupuncture and/or moxibustion. The values during and after stimulation of total HRV, of the low-frequency (LF) and high-frequency (HF), and those of LF/HF ratio should be compared to baseline values and values of healthy volunteers. Together with the results of the preliminary study, the present results can serve as a solid basis for further development of acupuncture stimulation and moxibustion equipment for complementary use in treating fatigue and symptoms of stress. Both institutions have experts for this kind of experiments.

\section{Keywords}

acupuncture, lifestyle diseases, high-tech acupuncture, Sino-Austrian cooperation, heart rate variability, evidence-based complementary medicine 


\section{Einleitung}

\section{Zusammenarbeit zwischen der Medizinischen Universität Graz und der Hubei-Universität für TCM in Wuhan}

Die Medizinische Universität Graz arbeitet im Rahmen des TCM-Forschungszentrums seit vielen Jahren intensiv mit der Hubei-Universität für Chinesische Medizin in Wuhan in China zusammen. Mit den höchsten VertreterInnen dieser Universität wurden bereits zahlreiche Projekte gemeinsam erfolgreich bewerkstelligt.

Die Hubei-Universität für Chinesische Medizin (Abb. 1), die im Jahr 1958 gegründet wurde, verfügt über 4 angeschlossene Spitäler, 4 medizinische Forschungszentren und 10 Forschungsinstitutionen. Das Universitätsgelände erstreckt sich über $0,65 \mathrm{~km}^{2}$, wovon rund 470.000 $\mathrm{m}^{2}$ als Arbeitsfläche zurVerfügung stehen. Die Universität hat 15 Abteilungen; sie bietet Studien für Bachelor- und Master-Grade sowie Doktoratsstudien an. Darüber hinaus finden sich unter ihrem Dach über 60 klinische Ausbildungsstationen an weiteren 6 angeschlossenen Spitälern und 21 Unterrichtslaboratorien. Zurzeit besuchen rund 15.000 Studierende diese Universität.

Akupunktur ist eine der Hauptforschungsdisziplinen dieser renommierten Institution. Das Team von Gerhard Litscher an der MedUni Graz arbeitet im Bereich der HighTech-Akupunkturforschung eng mit der Hubei-Universität für Chinesische Medizin (Prof. Wang Hua, ehemaliger Präsident der Universität und Prof. Liang Fengxia, Direktorin des Instituts für Akupunktur und Moxibustion) zusammen (Abb. 2). In den letzten Jahren wurden gemeinsame Arbeiten zur Veröffentlichung beispielsweise in Journalen der Nature-Gruppe angenommen [1-7]. Gerhard

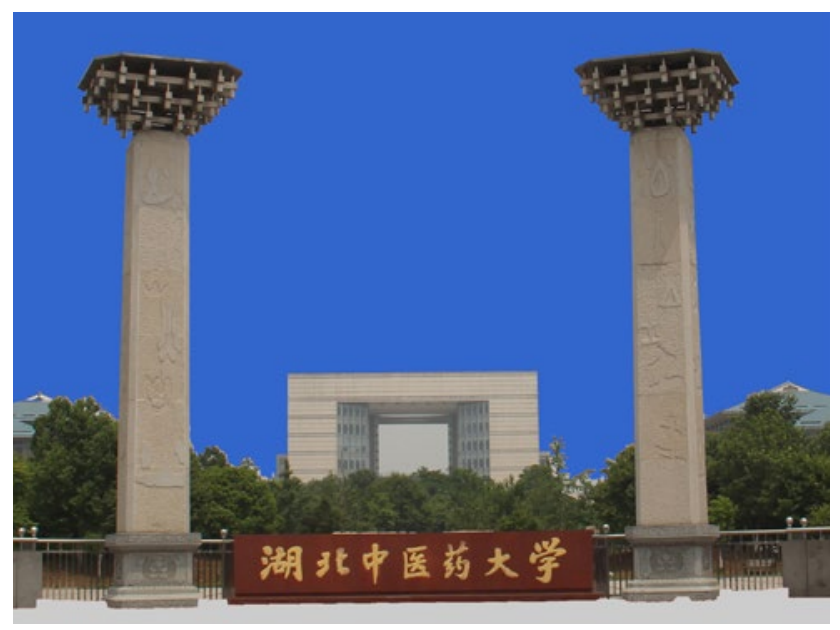

Abb. 1: Hubei Universität für Traditionelle Chinesische Medizin in Wuhan, China
Litscher ist darüber hinaus Gastprofessor an der HubeiUniversity of Chinese Medicine und am Hubei Provincial Collaborative Innovation Center of Preventive Treatment by Acupuncture \& Moxibustion. Prof. Hua Wang, der ehemalige langjährige Präsident dieser Universität, fördert die Zusammenarbeit zwischen Graz und Wuhan (Abb. 2).

Auch mit den RepräsentantInnen dieser renommierten Universität in Wuhan einigte man sich darauf, die bestehende Zusammenarbeit weiter zu intensivieren und gemeinsame Projekte neu zu initiieren bzw. fortzusetzen.

Ebenfalls während der COVID-19-Krise wurde seitens des Österreichischen Bundesministeriums für Bildung, Wissenschaft und Forschung und des OEAD ein von G. Litscher eingereichtes zweites „Sino-Austrian“ Projekt für die weitere Zusammenarbeit mit dem Titel „High-Tech Akupunktur für die Prävention von Lebensstil bedingten Erkrankungen - Eine ,Sino-Austrian' Kooperation - Fortsetzung" genehmigt (Dauer wie beim ersten Projekt: 1.7.2020 bis vorläufig 28.2.2022). Auch hier dürfte bei der Entscheidungsfindung der Gutachter des Ministeriums die Tatsache, dass es sich dabei um eine laufende „Tele-Akupunkturstudie“ in der Corona-Krisenzeit handelt, eine Rolle gespielt haben.

Müdigkeit und Stress, die durch längeres Training hervorgerufen werden, führen nicht nur zu einerVerringerung der Trainingskapazität, sondern können auch die Ursache vieler Krankheiten sein.Akupunktur kann zur Behandlung von Ermüdung bzw. Stress angewendet werden. Mit innovativen Methoden wie neuen Analysen der Herzratenvariabilität (HRV) können Krankheiten wie Belastungsermüdung untersucht werden. Das Hauptziel dieses Projekts ist die Erforschung der Beziehung zwischen Übungsermüdungssyndrom und Herzrate (HR) bzw. HRV.

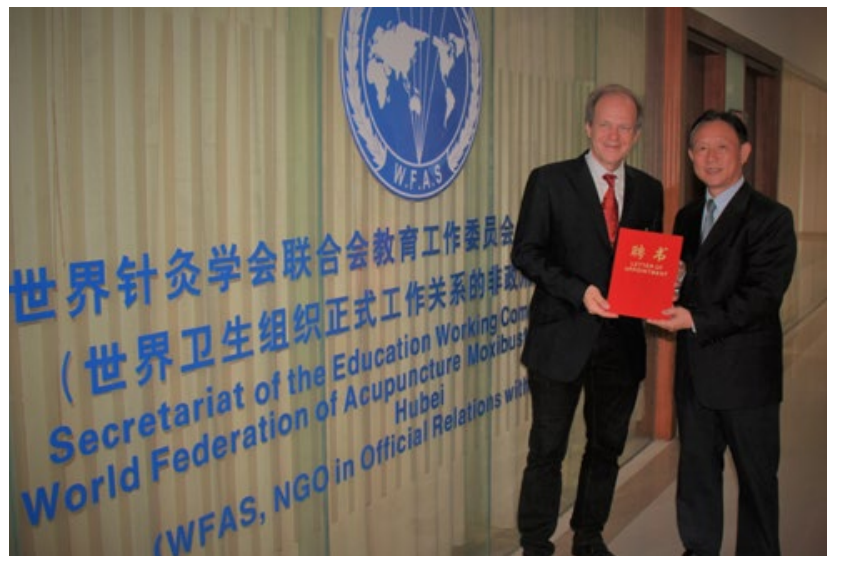

Abb. 2: Ehemaliger Präsident Prof. Hua Wang von der Hubei-Universität für Traditionelle Chinesische Medizin, Wuhan, China (rechts) und Prof. Gerhard Litscher von der Medizinischen Universität Graz noch vor der Corona-Krise in Wuhan 
PatientInnen mit körperlicher Müdigkeit und gesunde Freiwillige werden untersucht. Basierend auf Zufallszahlen, die vom Computer generiert und in undurchsichtigen Umschlägen verschlossen werden, werden die PatientInnen in eine Gruppe mit Ma-36-Akupunkturstimulation ( $\mathrm{Zu}$ sanli), eine Gruppe mit KG-4-Stimulation (Guanyuan), eine Gruppe mit beiden Punkten, eine Gruppe mit beiden Punkten, aber unterteilt ohne manuelle Nadelstimulation und eine Moxibustionsgruppe unterVerwendung der beiden o.g. Punkte eingeteilt. Insgesamt sind 4 verschiedene Experimente geplant:

- Experiment 1 ist ein Vergleich zwischen einer Übungsermüdungsgruppe und einer Kontrollgruppe (gesunde Freiwillige). Die Bewertungsparameter sind verschiedene Scores und Indizes von HR und HRV.

- Experiment 2 vergleicht die Wirksamkeit verschiedener Akupunkturpunkte.

- In Experiment 3 sollten Akupunktur und Moxibustion mit gesunden Freiwilligen verglichen werden.

- Experiment 4 untersucht die langfristigen therapeutischen Wirkungen von Akupunktur und Moxibustion auf die Scores von Belastungsermüdung und HR/HRV bei PatientInnen mit Belastungsermüdung.

Sowohl in der Akupunktur- als auch in der Moxibustionsgruppe werden insgesamt 10 Behandlungen durchgeführt.

Die gemeinsame transkontinentale Forschung zielt auf die wissenschaftliche Bewertung der Ermüdung bei körperlicher Betätigung ab, wobei in erster Linie die HRV bewertet werden soll. Dieser Parameter ist ein sehr guter Indikator für den Gesundheitszustand und kann durch verschiedene Akupunkturmethoden beeinflusst werden.

Das Forschungsprojekt wurde von der Ethikkommission des Hubei Provincial Hospital für Traditionelle Chinesische Medizin (TCM) mit der Zertifikatsnummer HBZY2016-C24-01 genehmigt. Es wurde auch auf ClinicalTrials.gov (ID NCT02924831) registriert.

Der erste Schritt bestand darin, PatientInnen per Poster und über soziale Medien (WeChat) zu rekrutieren. Dann begannen die ersten Testmessungen.

Wichtige Vorarbeiten wurden bereits 2019 durchgeführt [7]: So wurde bereits ein möglicher Unterschied zwischen den Auswirkungen von Akupunktur und Moxibustion - Guanyuan (KG 4) und Zusanli (Ma 36) - auf die unmittelbare Auswirkung der HRV beim Qi-Mangel-Syndrom analysiert. Dazu wurden insgesamt 90 PatientInnen mit Qi-Mangel zufällig in 3 Gruppen eingeteilt. Die Akupunkturgruppe wurde mit Akupunktur behandelt, die Moxibustionsgruppe wurde mit Moxibustion stimuliert und die Kontrollgruppe erhielt keine Intervention (insgesamt je 30 Personen). Die Veränderungen der Symptomwerte des Qi-Mangel-Syndroms wurden in jeder Gruppe vor und nach der Behandlung beobachtet. Die Veränderungen der HRV-bezogenen Indikatoren zu 5 Zeitpunkten vor der Behandlung, 5 Minuten, 10 Minuten, $15 \mathrm{Mi}-$ nuten während Behandlung sowie 5 Minuten nach der Behandlung wurden erfasst und aufgezeichnet.

Es gab keinen signifikanten Unterschied in den HRVbezogenen Indizes zwischen der Kontrollgruppe und den Behandlungsgruppen vor und während der Behandlung $(p>0,05)$. Die Akupunkturgruppe und die Moxibustionsgruppe wurden ebenfalls verglichen. Die mittleren HRV-Werte waren 10 min und 15 min nach der Behandlung signifikant niedriger $(\mathrm{p}<0,01)$; der Unterschied in der Gesamt-HRV zwischen der Akupunkturgruppe und der Moxibustionsgruppe nach 15 Minuten war statistisch signifikant $(\mathrm{p}<0,01)$. In der Akupunkturgruppe war der LF-Wert nach 5 Minuten signifikant erhöht $(\mathrm{p}<0,01)$ und die Werte der HR der Akupunkturgruppe und der Moxibustionsgruppe waren verringert $(p<0,05)$. Der LF/ HF-Wert der Akupunkturgruppe zeigte nach 5 Minuten und nach der Behandlung einen signifikanten Aufwärtstrend $(p<0,01)$, und der Unterschied der LF/HF-Werte in der Akupunktur- und Moxibustionsgruppe nach $10 \mathrm{Mi}-$ nuten war statistisch signifikant $(\mathrm{p}<0,05)$. Im Vergleich zur Kontrollgruppe war der Anstieg des gesamt HRVWertes in der Akupunkturgruppe signifikant $(\mathrm{p}<0,05)$ und der LF-Wert in der Akupunkturgruppe war signifikant verringert $(p<0,05)$. Der LF/HF-Wert nach der Behandlung zeigte einen signifikanten Aufwärtstrend ( $<<0,01)$.

Somit könnte gefolgert werden, dass die Akupunktur und Moxibustionstherapie einen gewissen unmittelbaren Einfluss auf die HRV beim Qi-Mangel-Syndrom haben, aber die Akupunkturgruppe zeigte mehr Verbesserungen als die Moxibustionsgruppe, was auf die Anpassungsoption des autonomen Nervensystems zurückzuführen sein könnte [7].

In unserem Projekt haben wir eine „Akupunkturgruppe ohne Stimulation“, die der „Akupunktur-Kompatibilitätsgruppe“ ähnlich ist. Um die Wirkung der PlaceboAkupunktur zu objektivieren, werden wir die sog. „Streitberger-Nadel“" verwenden, welche aus 3 Teilen besteht: skalierbare Nadel, Kunststoffring und Kunststoffabdeckung (Abb. 3). Diese Nadeln sind skalierbar und dringen daher nicht in die Haut ein (Abb. 4) [8].

Die „Streitberger-Nadel“ ist in der Akupunkturforschung anerkannt und wird seit der Erstvorstellung von Prof. Konrad Streitberger im Jahr 1998 in Graz erfolgreich eingesetzt [8]. 


\section{Konklusion}

Im Rahmen dieses Forschungsprojekts (Juli 2020 - Februar 2022) wollen wir unsere gemeinsame umfangreiche Studie in Wuhan abschließen, die Datenanalyse in Graz durchführen und weitere gemeinsameVeröffentlichungen für die wissenschaftliche Gemeinschaft von Wuhan und Graz aus vorbereiten.

\section{Danksagung}

In Österreich wird die Arbeit vom Eurasia Pacific Uninet (EPU-Projekt 36/2019; ,,High-Tech Akupunktur für die Prävention von Lebensstil bedingten Erkrankungen - Eine ,Sino-Austrian“ Kooperation - Fortsetzung “; Projektleiter G. Litscher) unterstützt.

\section{Beteiligte ForscherInnen}

Univ.-Prof. DI DDr. Gerhard Litscher (Projektleiter, Medizinische Universität Graz, Gastprofessor an der HubeiUniversität für TCM Wuhan, China), Priv.-Doz. Dr.med. Lu Wang (Medizinische Universität Graz), Prof. Hua Wang (ehem. Präsident der Hubei-Universität für TCM, Wuhan, China), Prof. Fengxia Liang (Direktorin des Instituts für Akupunktur und Moxibustion, Hubei-Universität für TCM Wuhan, China), Jingzhi Wang PhD, Li Chen PhD, Song Wu PhD Assoc. Prof., Jianmin Liu PhD Assoc. Prof.,

\section{Literatur}

[1] Litscher G: Modernization of acupuncture - research progress. Journal of Hubei University of Chinese Medicine 2015; 17(3): $1-6$.

[2] Shu Q, Wang H, Litscher D et al.: Acupuncture and moxibustion have different effects on fatigue by regulating the autonomic nervous system:A pilot controlled clinical trial. Sci Rep 2016; 6:37846. doi:10.1038/srep37846.

[3] Shu Q, Sun D, Wang H et al.: Differences of acupuncture and moxibustion on heart rate variability in qi-deficiency syndrome: a randomized controlled trial. Zhongguo Zhen Jiu 2017; 37: 25-30.

[4] Liang FX, Chen ZB, Wu S et al.: High-tech acupuncture for prevention of lifestyle diseases - a Sino-Austrian cooperation research protocol on heart rate variability. Chin J Integr Med 2018; 24: 423-428. doi:10.1007/s11655-017-2548-4.

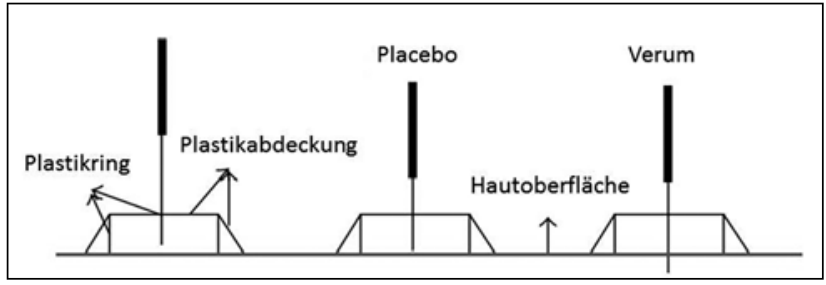

Abb. 3: Schematisierte Wirkungsweise der „Streitberger-Nadel“ zur Placebo-Akupunktur
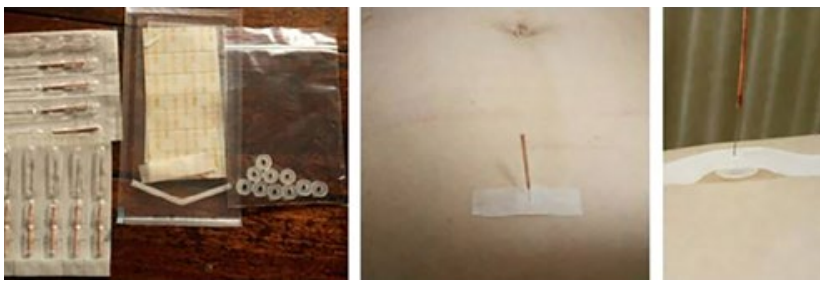

Abb. 4: „Streitberger-Nadel“ in der praktischen Anwendung des gegenständlichen Projekts

Qing Shu PhD, Qi Huang PhD cand., Yayuan Wang PhD cand.,Yanjuan Song PhD,Yudian Zhouz MSc cand., Tong Li MSc cand., Jinxiao MSc cand., Yue Li, Xianglin Chen, Lingling Shi, Yujie Li (alle Hubei-Universität für TCM Wuhan, China).

Interessenkonflikt: Die Autoren erklären keinen Interessenkonflikt.

[5] Litscher G, Liang FX: Qigong and health care prevention special aspects from the Chinese perception (in German). Akupunkt \& Aurikulomed 2017; 43: 26-30.

[6] Liang FX, Huang Q, Chen R et al.: Healthcare-related qigong for the prevention of lifestyle-related diseases. OBM ICM 2017; 2: 002. doi:10.21926/obm.icm.1702002.

[7] Song YY, Liang FX, Wang $\mathrm{H}$ et al.: Effect of acupuncture and moxibustion on tonicity points of heart rate variability of qideficiency syndrome. Zhongguo Zhen Jiu 2020; in press.

[8] Streitberger K, Kleinhenz J. Introducing a placebo needle into acupuncture research. Lancet 1998;352:364-365. doi:10.1016/ S0140-6736(97)10471-8. 